\title{
Diffusion Processes Satisfying a Conservation Law Constraint
}

\author{
J. Bakosi and J. R. Ristorcelli \\ Los Alamos National Laboratory, Los Alamos, NM 87545, USA \\ Correspondence should be addressed to J. Bakosi; jbakosi@lanl.gov \\ Received 12 November 2013; Accepted 5 January 2014; Published 4 March 2014 \\ Academic Editor: Nikolai Leonenko
}

Copyright (C) 2014 J. Bakosi and J. R. Ristorcelli. This is an open access article distributed under the Creative Commons Attribution License, which permits unrestricted use, distribution, and reproduction in any medium, provided the original work is properly cited.

\begin{abstract}
We investigate coupled stochastic differential equations governing $N$ nonnegative continuous random variables that satisfy a conservation principle. In various fields a conservation law requires a set of fluctuating variables to be nonnegative and (if appropriately normalized) sum to one. As a result, any stochastic differential equation model to be realizable must not produce events outside of the allowed sample space. We develop a set of constraints on the drift and diffusion terms of such stochastic models to ensure that both the nonnegativity and the unit-sum conservation law constraints are satisfied as the variables evolve in time. We investigate the consequences of the developed constraints on the Fokker-Planck equation, the associated system of stochastic differential equations, and the evolution equations of the first four moments of the probability density function. We show that random variables, satisfying a conservation law constraint, represented by stochastic diffusion processes, must have diffusion terms that are coupled and nonlinear. The set of constraints developed enables the development of statistical representations of fluctuating variables satisfying a conservation law. We exemplify the results with the bivariate beta process and the multivariate Wright-Fisher, Dirichlet, and Lochner's generalized Dirichlet processes.
\end{abstract}

\section{Introduction and Problem Statement}

We investigate the consequences of the unit-sum requirement on $N>1$ nonnegative continuous random variables governed by a diffusion process. Such mathematical description is useful to represent fluctuating variables, $Y_{1}, \ldots, Y_{N}$, subject to the constraint $\sum Y_{\alpha}=1$. We are interested in stochastic diffusion models and statistical moment equations describing the temporal evolutions $Y_{\alpha}=Y_{\alpha}(t)$ and their statistics. In particular, we study the consequences of the bounded sample space, required by the nonnegativity of $Y_{\alpha}$ and the unit-sum conservation principle, $\sum Y_{\alpha}=1$. A simple physical example is the mixture of different chemical species, represented by mass fractions $0 \leq Y_{\alpha} \leq 1$ undergoing reaction in a fluid whose overall mass is conserved. Such mathematical problems also appear in evolutionary theory [1], Bayesian statistics [2], geology [3-5], forensics [6], econometrics [7], turbulent mixing and combustion [8], and population biology [9]. Mathematical properties of such random fractions are given in [10-13].
Mathematically, we are interested in the following question. What functions are allowed to represent the drift, $A_{\alpha}$, and diffusion, $b_{\alpha \beta}$, terms of the system, governing the vector $\mathbf{Y}=\left(Y_{1}, \ldots, Y_{N}\right)$

$$
\begin{array}{r}
\mathrm{d} Y_{\alpha}(t)=A_{\alpha}(\mathbf{Y}, t) \mathrm{d} t+\sum_{\beta=1}^{N} b_{\alpha \beta}(\mathbf{Y}, t) \mathrm{d} W_{\beta}(t), \\
\alpha=1, \ldots, N,
\end{array}
$$

if

$$
Y_{\alpha} \geq 0, \quad \alpha=1, \ldots, N, \quad \sum_{\alpha=1}^{N} Y_{\alpha}=1
$$

must hold for all $t$. In (1) $\mathrm{d} W_{\alpha}(t)$ is a vector-valued Wiener process with mean $\left\langle\mathrm{d} W_{\alpha}\right\rangle=0$ and covariance $\left\langle\mathrm{d} W_{\alpha} \mathrm{d} W_{\beta}\right\rangle=$ $\delta_{\alpha \beta} \mathrm{d} t$; see [14], and $\delta_{\alpha \beta}$ is Kronecker's delta. If the components of $\mathbf{Y}$ satisfy the constraints in (2), we call the event $\mathbf{Y}$ 
realizable. A consequence of the constraints in (2) imposed on the stochastic system (1) is that for all $t$ the following holds:

$$
\begin{aligned}
\sum_{\alpha=1}^{N} \mathrm{~d} Y_{\alpha}(t)=0= & \sum_{\alpha=1}^{N} A_{\alpha}(\mathbf{Y}, t) \mathrm{d} t \\
& +\sum_{\alpha=1}^{N} \sum_{\beta=1}^{N} b_{\alpha \beta}(\mathbf{Y}, t) \mathrm{d} W_{\beta}(t) .
\end{aligned}
$$

In other words, we are interested in expressions for $A_{\alpha}$ and $b_{\alpha \beta}$, what constraints they must satisfy in addition to (3), and how to implement them so that (1) produces realizable events; that is, $\mathbf{Y}$ satisfies (2) for all $t$.

We study diffusion processes as (1) they are mathematically simple vehicles for representing temporal evolutions of fluctuating fractions (of a unit) and their statistics, (2) they lend themselves to simple Monte-Carlo numerical methods [15], and (3) they serve as a starting point for representations of statistical moment equations if individual samples and joint probabilities are not required. The Markovian assumption [14] is made at the outset and jump contributions are ignored. We derive constraints for the drift and diffusion terms that assure that the modeled processes are realizable (i.e., produce nonnegative variables that satisfy the unit-sum constraint) for any realization at all times. We address the problem of the functional forms of the drift and diffusion terms from three perspectives: (1) the FokkerPlanck equation for the probability density function, (2) the stochastic differential equations for the individual realizations, and (3) the evolution equations for the jointly coupled statistics.

The plan of the paper is as follows. Section 2 introduces the geometry of the multidimensional sample space within which realizations of fractions of a unit are allowed and discusses constraints that ensure realizable statistical moments. Section 3 develops the implications of realizability on diffusion processes governing fractions. Section 4 follows by developing realizability constraints on the time evolutions of statistics. Section 5 surveys some existing realizable diffusion processes. A summary is given in Section 6.

\section{Realizability due to Conservation}

The notion of realizability due to a conservation law constraint was introduced and defined by (2). We now discuss the consequences of realizability pertaining to the individual samples of the state space, Section 2.1, and of their statistics, Section 2.2.

2.1. The Universal Geometry of Allowed Realizations. The geometrical definition of the sample space is given in which the vector $\mathbf{Y}=\left(Y_{1}, \ldots, Y_{N}\right)$ is allowed if (2) is to be satisfied. This is used to derive constraints for stochastic diffusions and their moment equations in the subsequent sections.
A realization of the vector, $\mathbf{Y}$, with coordinates $Y_{\alpha} \geq 0, \alpha=$ $1, \ldots, N$, specifies a point in the multidimensional sample space. The union of all such points, that satisfy

$$
\sum_{\alpha=1}^{N} Y_{\alpha}=1
$$

is the space of allowed realizations; see (2). For example, in representing mass fraction constituents of a substance, (4) restricts the possible components of $\mathbf{Y}$ to those that are realizable; those vectors that point outside of the allowed space are not conserved; if (4) is violated, spurious mass is created or destroyed.

Mathematically, the geometry of allowed realizations is a simplex, the generalization of a triangle to multiple dimensions. For $N$ variables the $(N-1)$-simplex is a bounded convex polytope, $\mathscr{P}$, on the $(N-1)$-dimensional hyperplane; $\mathscr{P}$ is the convex hull of its $N$ vertices. $\mathscr{P}$ 's boundary, $\partial \mathscr{P}$, is defined as the closed surface of nonoverlapping hyperplanes of $N-2$ dimensions:

$$
\partial \mathscr{P} \equiv\left(Y_{\alpha}=0: \alpha=1, \ldots, N-1 ; \sum_{\alpha=1}^{N-1} Y_{\alpha}=1\right)
$$

plotted in Figure 1 for $N=3$.

The domain (or support) of the joint probability, $F(\mathbf{Y})$, with $Y_{\alpha}, \alpha=1, \ldots, N$, is the $(N-1)$-simplex. Of all $Y_{\alpha}$ only $N-1$ are independent due to (4) and without loss of generality we take

$$
Y_{N}=1-\sum_{\alpha=1}^{N-1} Y_{\alpha}
$$

The same geometry of allowed realizations is discussed by Pope in the $N$-dimensional state space in [16] in the context of ideal gas mixing in turbulent combustion.

We confine our attention here to $N-1$ dimensions, as one of the variables is determined by the unit-sum requirement; see (6). As a consequence, the $(N-1)$-dimensional geometry of realizable events is remarkably simple and universal: it is the bounded convex polytope whose boundary is defined by (5). Consequently, the realizability constraint, (2), uniquely and universally determines the realizable region of the state space: it is the same in all points in space and time for all materials undergoing any physical process that conserves mass; see (4). The ensemble is realizable if and only if all samples reside inside the polytope given by (5). For $N=3$ this means that the support of $F$ is the triangle depicted in Figure 1.

2.2. Realizable Statistical Moments. If the fractions are nonnegative and sum to one, required by (2), they are also bounded:

$$
0 \leq Y_{\alpha} \leq 1, \quad \alpha=1, \ldots, N, \quad \sum_{\alpha=1}^{N} Y_{\alpha}=1
$$

whose consequences on some of their statistical moments are now discussed. 


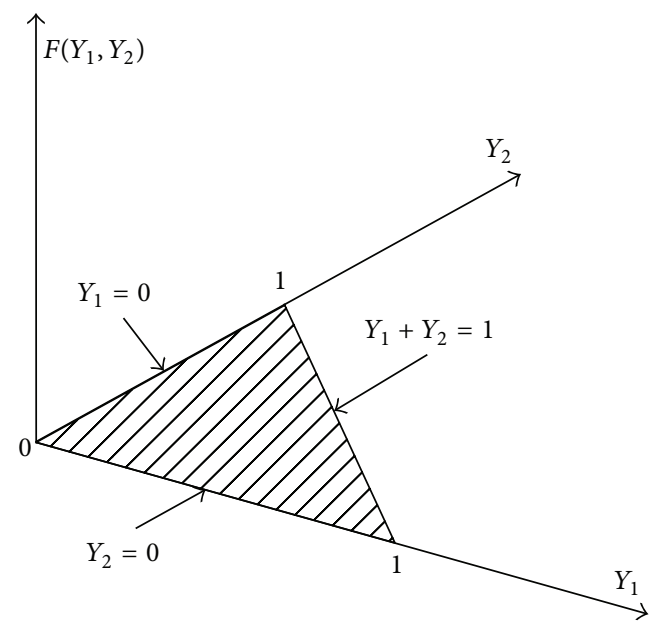

FIgURE 1: The geometry of allowed realizations for $N=3$ variables, $\mathbf{Y}=\left(Y_{1}, Y_{2}, Y_{3}=1-Y_{1}-Y_{2}\right)$, that satisfy nonnegativity and the unit-sum constraint; see (2). The boundary of the allowed region on the plane spanned by $Y_{1}$ and $Y_{2}$ is the closed loop of straight lines: $\left(Y_{1}=0, Y_{2}=0, Y_{1}+Y_{2}=1\right)$, defined by (5). If the vector $Y$ points inside the triangle, it is a realizable event.

Taking mathematical expectations of (7), see for example, [17] yields

$$
0 \leq\left\langle Y_{\alpha}\right\rangle \leq 1, \quad \alpha=1, \ldots, N, \quad \sum_{\alpha=1}^{N}\left\langle Y_{\alpha}\right\rangle=1
$$

Similar to the instantaneous fractions, the first statistical moments are also nonnegative, are bounded, and sum to unity.

Since both the instantaneous variables and their means are bounded, fluctuations about the means are also bounded:

$$
-1 \leq y_{\alpha}=Y_{\alpha}-\left\langle Y_{\alpha}\right\rangle \leq 1
$$

As a consequence, the variances and the covariances are also bounded:

$$
\begin{gathered}
0 \leq\left\langle y_{\alpha}^{2}\right\rangle=\left\langle\left(Y_{\alpha}-\left\langle Y_{\alpha}\right\rangle\right)^{2}\right\rangle \leq 1 \\
-1 \leq\left\langle y_{\alpha} y_{\beta}\right\rangle=\left\langle\left(Y_{\alpha}-\left\langle Y_{\alpha}\right\rangle\right)\left(Y_{\beta}-\left\langle Y_{\beta}\right\rangle\right)\right\rangle \leq 1, \quad \alpha \neq \beta .
\end{gathered}
$$

Multiplying (4) by $y_{\beta}, \beta=1, \ldots, N$ and taking the expectation yield

$$
\begin{gathered}
\left\langle y_{1}^{2}\right\rangle+\left\langle y_{2} y_{1}\right\rangle+\cdots+\left\langle y_{N} y_{1}\right\rangle=0 \\
\left\langle y_{1} y_{2}\right\rangle+\left\langle y_{2}^{2}\right\rangle+\cdots+\left\langle y_{N} y_{2}\right\rangle=0 \\
\vdots \\
\left\langle y_{1} y_{N}\right\rangle+\left\langle y_{2} y_{N}\right\rangle+\cdots+\left\langle y_{N}^{2}\right\rangle=0
\end{gathered}
$$

that is, the row sums and, due to symmetry, the column sums of the covariance matrix are zero. Expressing $\left\langle y_{N} y_{1}\right\rangle$, $\left\langle y_{N} y_{2}\right\rangle$, and so forth, from the first $N-1$ equations of (12), and substituting them into the $N$ th one yield the weaker constraint:

$$
\sum_{\alpha=1}^{N-1} \sum_{\beta=1}^{N-1}\left\langle y_{\alpha} y_{\beta}\right\rangle-\left\langle y_{N}^{2}\right\rangle=0
$$

Due to bounded fluctuations, see (9), the third central moments are also bounded:

$$
-1 \leq\left\langle y_{\alpha}^{3}\right\rangle=\left\langle\left(Y_{\alpha}-\left\langle Y_{\alpha}\right\rangle\right)^{3}\right\rangle \leq 1
$$

and in general, for $n \geq 2$ we have

$$
\begin{aligned}
& 0 \leq\left\langle y_{\alpha}^{n}\right\rangle \leq 1, \quad \text { for even } n, \\
& -1 \leq\left\langle y_{\alpha}^{n}\right\rangle \leq 1, \quad \text { for odd } n .
\end{aligned}
$$

Ensuring nonnegativity and unit sum puts constraints on possible time evolutions of $\mathbf{Y}=\mathbf{Y}(t)$, represented by diffusion processes and that of their statistics. Some of these constraints are developed in the following sections.

\section{Diffusion Processes for Random Fractions}

Implications of the geometry of the realizable state space, discussed in Section 2, on diffusion processes are developed. First, the relevant mathematical properties of Fokker-Planck equations are reviewed in Section 3.1, followed by the constraints on their functional forms, Section 3.2.

3.1. Review of Some Boundary Conditions of Fokker-Planck Equations. The discussion is restricted to Markov processes which by definition obey a Chapman-Kolmogorov equation [14]. Assuming that $Y_{\alpha}$ are continuous in space and time, jump processes are excluded. The temporal evolution of random fractions, $\mathbf{Y}(t)$, constrained by (2) can then be represented most generally by diffusion processes whose transitional probability, $F(\mathbf{Y}, t)$, is governed by the FokkerPlanck equation:

$$
\begin{aligned}
\frac{\partial}{\partial t} F(\mathbf{Y}, t)= & -\sum_{\alpha=1}^{N-1} \frac{\partial}{\partial Y_{\alpha}}\left[A_{\alpha}(\mathbf{Y}, t) F(\mathbf{Y}, t)\right] \\
& +\frac{1}{2} \sum_{\alpha=1}^{N-1} \sum_{\beta=1}^{N-1} \frac{\partial^{2}}{\partial Y_{\alpha} \partial Y_{\beta}}\left[B_{\alpha \beta}(\mathbf{Y}, t) F(\mathbf{Y}, t)\right],
\end{aligned}
$$

where $A_{\alpha}$ and $B_{\alpha \beta}$ denote drift and diffusion in state space, respectively, and $B_{\alpha \beta}$ is symmetric nonnegative semidefinite [17]. Equation (17) is a partial differential equation that governs the joint probability, $F(\mathbf{Y}, t)$, of the fractions, $Y_{\alpha}$, $\alpha=1, \ldots, N-1 . Y_{N}$ is excluded from (17) and is determined by (6). Augmented by initial and boundary conditions, (17) describes the transport of probability in sample space $\mathscr{R}$ whose boundary is $\partial \mathscr{R}$ with normal vector $n_{\alpha}$; see [14].

Equation (17) can be written in conservation form as

$$
\frac{\partial}{\partial t} F(\mathbf{Y}, t)+\sum_{\alpha=1}^{N-1} \frac{\partial}{\partial Y_{\alpha}} I_{\alpha}(\mathbf{Y}, t)=0,
$$


in terms of the probability flux; see [14, Section 5.1]:

$$
\begin{array}{r}
I_{\alpha}(\mathbf{Y}, t)=A_{\alpha}(\mathbf{Y}, t) F(\mathbf{Y}, t) \\
-\frac{1}{2} \sum_{\beta=1}^{N-1} \frac{\partial}{\partial Y_{\beta}}\left[B_{\alpha \beta}(\mathbf{Y}, t) F(\mathbf{Y}, t)\right], \\
\quad \alpha=1, \ldots, N-1 .
\end{array}
$$

Using (18) and (19) the following boundary conditions are considered; see [14, Section 6.2].

(1) Reflecting barrier. If $\sum n_{\alpha} I_{\alpha}(\mathbf{Y}, t)=0$ everywhere on the boundary, $\partial \mathscr{R}$ is a reflecting barrier: a particle inside $\mathscr{R}$ cannot cross the boundary and must be reflected there.

(2) Absorbing barrier. If $F(\mathbf{Y}, t)=0$ everywhere on the boundary, $\partial \mathscr{R}$ is an absorbing barrier: if a particle reaches the boundary, it is removed from the system.

(3) Other types of boundary conditions. Some part of the boundary may be reflecting while some other may be absorbing: a combination is certainly possible. We only consider reflecting and absorbing barriersother types of boundaries are discussed in [18].

To support the forthcoming discussion, some well-established mathematical properties of multivariable FokkerPlanck equations have been reviewed.

3.2. Realizable Diffusion Processes. The implications of the realizability constraint, (2), on the functional forms of the drift and diffusion terms of the Fokker-Planck equation (17) are now investigated.

As discussed in Section 2, the region of the sample space allowed by the realizability requirement is the polytope $\mathscr{P}$ defined by its boundary, $\partial \mathscr{P},(5)$, in which all samples of $\mathbf{Y}=$ $\mathrm{Y}(t)$ must reside at all times. Consequently, the sample space, $\mathscr{R}$, of the Fokker-Planck equation (17) must coincide with $\mathscr{P}$, which constrains the possible functional forms of $A_{\alpha}(\mathbf{Y}, t)$ and $B_{\alpha \beta}(\mathbf{Y}, t)$. In the following, these constraints are developed for binary (single-variable) processes first, followed by ternary processes, and then generalized to multiple variables.

3.2.1. Realizable Binary Processes: $N=2$. The Itô diffusion process [14], governing the variable $Y$,

$$
\mathrm{d} Y(t)=A(Y, t) \mathrm{d} t+\sqrt{B(Y, t)} \mathrm{d} W(t),
$$

with $B(Y, t) \geq 0$ is equivalent to and derived from (17) with $N=2$; see for example, [14]:

$$
\frac{\partial}{\partial t} F(Y, t)=-\frac{\partial}{\partial Y}[A(Y, t) F(Y, t)]+\frac{1}{2} \frac{\partial^{2}}{\partial Y^{2}}[B(Y, t) F(Y, t)]
$$

For $N=2$ the allowed space of realizations is a line with endpoints given by (5):

$$
(Y=0 ; \quad Y=1)
$$

This can be ensured if the drift and diffusion terms in (20) and (21) satisfy

$$
\begin{array}{ll}
A(Y=0, t) \geq 0, & B(Y=0, t)=0, \\
A(Y=1, t) \leq 0, & B(Y=1, t)=0 .
\end{array}
$$

In other words, the realizability constraint in (2) on (20) mathematically corresponds to (23). A diffusion process, governed by (20), that satisfies (23), ensures that the fractions $Y$ and $1-Y$ satisfy $0 \leq Y \leq 1$, provided each event of the ensemble at $t=0$ satisfies $0 \leq Y \leq 1$. The equal signs in the constraints on the drift in (23) allow for absorbing barriers at $Y=0$ and $Y=1$, respectively. The constraints on the diffusion term imply that $B(Y, t)$ must either be nonlinear in $Y$ or $B(Y, t) \equiv 0$ for all $Y$. In other words, since the diffusion term must be nonnegative, required by (20), it can only be nonzero inside the allowed sample space if it is also nonlinear.

3.2.2. Realizable Ternary Processes: $N=3$. For $N=3$ variables, the unit-sum-constrained sample space and its boundary are sketched in Figure 1. In this case individual samples of the joint probability, $F\left(Y_{1}, Y_{2}\right)$, are governed by the system:

$$
\begin{aligned}
\mathrm{d} Y_{1}(t)= & A_{1}\left(Y_{1}, Y_{2}, t\right) \mathrm{d} t+b_{11}\left(Y_{1}, Y_{2}, t\right) \mathrm{d} W_{1}(t) \\
& +b_{12}\left(Y_{1}, Y_{2}, t\right) \mathrm{d} W_{2}(t) \\
\mathrm{d} Y_{2}(t)= & A_{2}\left(Y_{1}, Y_{2}, t\right) \mathrm{d} t+b_{21}\left(Y_{1}, Y_{2}, t\right) \mathrm{d} W_{1}(t) \\
& +b_{22}\left(Y_{1}, Y_{2}, t\right) \mathrm{d} W_{2}(t) .
\end{aligned}
$$

The allowed samples space is two dimensional (a triangle) whose boundary, defined by (5), consists of the loop of lines:

$$
\left(Y_{1}=0, Y_{2}=0, Y_{1}+Y_{2}=1\right)
$$

For $N=3$, the state vector, governed by (24) augmented by $Y_{3}=1-Y_{1}-Y_{2}$, stays inside the allowed region if

$$
\begin{gathered}
A_{1}\left(Y_{1}=0, Y_{2}, t\right) \geq 0, \\
B_{11}\left(Y_{1}=0, Y_{2}, t\right)=B_{12}\left(Y_{1}=0, Y_{2}, t\right)=0, \\
A_{2}\left(Y_{1}, Y_{2}=0, t\right) \geq 0, \\
B_{21}\left(Y_{1}, Y_{2}=0, t\right)=B_{22}\left(Y_{1}, Y_{2}=0, t\right)=0, \\
A_{1}\left(Y_{1}+Y_{2}=1, t\right) \leq 0, \\
B_{11}\left(Y_{1}+Y_{2}=1, t\right)=B_{12}\left(Y_{1}+Y_{2}=1, t\right)=0, \\
A_{2}\left(Y_{1}+Y_{2}=1, t\right) \leq 0, \\
B_{21}\left(Y_{1}+Y_{2}=1, t\right)=B_{22}\left(Y_{1}+Y_{2}=1, t\right)=0 .
\end{gathered}
$$

The realizability constraint, (2), on the system of (24) mathematically corresponds to (26). The three fractions, $Y_{1}, Y_{2}$, and $Y_{3}=1-Y_{1}-Y_{2}$, governed by (6) and (24), remain fractions of unity if their drift and diffusion terms satisfy (26). Naturally, an initial ensemble that satisfies $0 \leq Y_{1}, 0 \leq Y_{2}$, and $Y_{1}+Y_{2} \leq 1$ 
is required. The constraints on the diffusion terms in (26) show that both $B_{1}$ and $B_{2}$ must either be nonlinear in $Y_{1}$ and $Y_{2}$, respectively, or $B_{1}\left(Y_{1}, t\right) \equiv 0$ and $B_{2}\left(Y_{2}, t\right) \equiv 0$, for all $Y_{1}$ and $Y_{2}$, respectively. Furthermore, if one were to construct a process with $A_{1}=A_{1}\left(Y_{1}\right), B_{11}=B_{11}\left(Y_{1}\right)$, and $B_{12}=B_{12}\left(Y_{1}\right)$, then either $A_{2}$ or $B_{22}$ must be a function of both $Y_{1}$ and $Y_{2}$ if $1-Y_{1}-Y_{2} \geq 0$ is to be maintained, required by $Y_{1}+Y_{2}+Y_{3}=1$ with $Y_{1} \geq 0, Y_{2} \geq 0, Y_{3} \geq 0$. In other words, the unit-sum constraint couples at least 2 of the 3 fractions, governed by the system given by (6) and (24).

3.2.3. Realizable Multi-Variable Processes: $N>2$. The multivariate Itô diffusion process, equivalent to the Fokker-Planck equation (17), is [14]

$$
\begin{array}{r}
\mathrm{d} Y_{\alpha}(t)=A_{\alpha}(\mathbf{Y}, t) \mathrm{d} t+\sum_{\beta=1}^{N-1} b_{\alpha \beta}(\mathbf{Y}, t) \mathrm{d} W_{\beta}(t), \\
\alpha=1, \ldots, N-1,
\end{array}
$$

with $B_{\alpha \beta}=\sum_{\gamma=1}^{N-1} b_{\alpha \gamma} b_{\gamma \beta}$ and the vector-valued Wiener process, $\mathrm{d} W_{\beta}(t)$, with mean $\left\langle\mathrm{d} W_{\beta}\right\rangle=0$ and covariance $\left\langle\mathrm{d} W_{\alpha} \mathrm{d} W_{\beta}\right\rangle=\delta_{\alpha \beta} \mathrm{d} t$. The sample space of allowed realizations is now bounded by the nonoverlapping hyperplanes, defined by (5). The conditions, analogous to (23) and (26) that ensure realizability for multiple variables, are

$$
\begin{gathered}
A_{\alpha}\left(Y_{\alpha}=0, Y_{\beta \neq \alpha}, t\right) \geq 0, \\
B_{\alpha \beta}\left(Y_{\alpha}=0, Y_{\beta \neq \alpha}, t\right)=0, \\
A_{\alpha}\left(\sum_{\alpha=1}^{N-1} Y_{\alpha}=1, t\right) \leq 0, \\
B_{\alpha \beta}\left(\sum_{\alpha=1}^{N-1} Y_{\alpha}=1, t\right)=0, \\
\alpha, \beta=1, \ldots, N-1 .
\end{gathered}
$$

The realizability constraint in (2) on the system of (27) mathematically corresponds to (28). A diffusion process, governed by (27), that satisfies (28) ensures that the fractions $Y_{\alpha}$ satisfy $0 \leq Y_{\alpha} \leq 1, \alpha=1, \ldots, N$, with $Y_{N}=1-$ $\sum_{\beta=1}^{N-1} Y_{\beta}$, provided each event of the initial ensemble at $t=0$ satisfies $0 \leq Y_{\alpha} \leq 1$. As before, the equal signs in the constraints on the drifts in (28) allow for absorbing barriers at the boundaries. The constraints on the diffusion term imply that for any $\alpha, B_{\alpha \beta}\left(Y_{\alpha}, Y_{\beta \neq \alpha}, t\right)$ must either be nonlinear in $Y_{\alpha}$ or $B_{\alpha \beta}\left(Y_{\alpha}, Y_{\beta \neq \alpha}, t\right) \equiv 0$ for all $Y_{\alpha}$. In other words, since the diffusion term must be nonnegative semidefinite, required by (27), it can only be nonzero inside the allowed sample space if it is also nonlinear. Equations (28) also show, that while it is conceivable, that $A_{\alpha} \neq A_{\alpha}\left(Y_{\beta}\right)$ and $B_{\alpha \beta} \neq B_{\alpha \beta}\left(Y_{\beta}\right)$ for a single $\alpha$ and all $\beta \neq \alpha$, if $\sum Y_{\alpha}=1$ is to be satisfied, either $A_{\alpha}=A_{\alpha}\left(Y_{\beta}\right)$ or $B_{\alpha \beta}=B_{\alpha \beta}\left(Y_{\beta}\right)$ must hold for all $\beta \neq \alpha$. In other words, the unit-sum constraint couples at least $N-1$ equations of the system of (6) and (27) governing $Y_{\alpha}$, $\alpha=1, \ldots, N$.
Constraints on the functional forms of the drift and diffusion terms of the multivariate Fokker-Planck equation (17), as a temporal representation of random fractions, $Y_{\alpha}=$ $Y_{\alpha}(t)$, have been developed. Equations (28) are our central result which ensure that sample space events, generated by (17) or its equivalent system of diffusion processes, (27), satisfy the realizability constraint at all times, provided the initial ensemble is realizable. Since (17) and (27) govern $N-1$ variables and $Y_{N}=1-\sum_{\beta=1}^{N-1} Y_{\beta}$, the unit-sum requirement, (4), is satisfied at all times. An implication of (28), exemplified in Section 5 , is that random fractions represented by diffusion processes must be coupled and nonlinear.

\section{Realizable Evolution of Statistics}

Some implications of (28) for the first few statistical moments of the joint probability, governed by (17), are now derived. This is useful for statistical moment equation representation of fractions if individual samples and joint probabilities are not required.

4.1. Realizable Evolution of the Means: $\left\langle Y_{\alpha}\right\rangle$. Multiplying (17) by $Y_{\gamma}$ and integrating over all sample space, see for example [19], yield the system of equations governing the means of the fractions:

$$
\frac{\partial\left\langle Y_{\alpha}\right\rangle}{\partial t}=\left\langle A_{\alpha}\right\rangle=\mathscr{M}_{\alpha}, \quad \alpha=1, \ldots, N-1,
$$

where $A_{\alpha}=A_{\alpha}(\mathbf{Y}, t)$. The evolution of the means can be made consistent with the realizability constraint in (2) if the means are bounded and sum to one at all times. Equation (29) shows that to keep the means bounded, required by (8), the rate of change of the means, $\mathscr{M}_{\alpha}$, must be governed by functions that satisfy

$$
\begin{aligned}
& \lim _{\left\langle Y_{\alpha}\right\rangle \rightarrow 0} \mathscr{M}_{\alpha}=\lim _{\left\langle Y_{\alpha}\right\rangle \rightarrow 0}\left\langle Y_{\alpha}\right\rangle_{{ }_{t}} \geq 0, \\
& \lim _{\left\langle Y_{\alpha}\right\rangle \rightarrow 1} \mathscr{M}_{\alpha}=\lim _{\left\langle Y_{\alpha}\right\rangle \rightarrow 1}\left\langle Y_{\alpha}\right\rangle_{{ }_{t}} \leq 0,
\end{aligned}
$$

as the boundary of the state space is approached. In (30) $(\cdot)_{t}=\partial / \partial t$. Equation (30) implies that inside the state space (i.e., away from the boundaries) $\mathscr{M}_{\alpha}$ must either be a function of $\left\langle Y_{\alpha}\right\rangle$ or $\mathscr{M}_{\alpha} \equiv 0$ for all $t$. The means may also sum to one, required by (8), if at least $N-2$ of (29) are coupled to each other. Consequently, $\mathscr{M}_{\alpha}$ must be a function of $\left\langle Y_{\beta}\right\rangle$ for all $\beta \neq \alpha$. Equation (29) shows how the means are governed if a Fokker-Planck equation (17) or a diffusion process (27) governs the underlying joint probability; for example, only the mean of the drift, $A_{\alpha}$, affects the evolution of the means.

4.2. Realizable Evolution of the Second Central Moments: $\left\langle y_{\alpha} y_{\beta}\right\rangle$. Multiplying the Fokker-Planck equation (17) by $y_{\gamma} y_{\delta}=\left(Y_{\gamma}-\left\langle Y_{\gamma}\right\rangle\right)\left(Y_{\delta}-\left\langle Y_{\delta}\right\rangle\right)$ and then integrating over 
all sample space yield the equations governing the covariance matrix of the fractions:

$$
\begin{array}{r}
\frac{\partial\left\langle y_{\alpha} y_{\beta}\right\rangle}{\partial t}=\left\langle y_{\alpha} A_{\beta}\right\rangle+\left\langle y_{\beta} A_{\alpha}\right\rangle+\left\langle B_{\alpha \beta}\right\rangle=\mathscr{C}_{\alpha \beta}, \\
\alpha, \beta=1, \ldots, N-1,
\end{array}
$$

with $A_{\alpha}=A_{\alpha}(\mathbf{Y}, t)$ and $B_{\alpha \beta}=B_{\alpha \beta}(\mathbf{Y}, t)$. The right hand side of (31) is denoted by $\mathscr{C}_{\alpha \beta}$, the evolution rate of the covariance matrix. Equation (31) shows how the covariances are governed if a Fokker-Planck equation (17) or a diffusion process (27) governs the underlying joint probability; for example, $\left\langle y_{\alpha} y_{\beta}\right\rangle$ is symmetric at all times. Following the development in Section 2.2, four conditions must be satisfied by the system of second moment equations (31) to ensure an evolution of the covariances that is consistent with the realizability constraint in (2).

(1) Symmetric covariance evolution. The symmetry of the covariance matrix can be ensured if $\left\langle y_{\alpha} y_{\beta}\right\rangle(t=0)$ is symmetric, as well as its evolution rates:

$$
\mathscr{C}_{\alpha \beta}=\mathscr{C}_{\beta \alpha} \text {. }
$$

(2) Boundedness of the variances, (10). This condition can be ensured with

$$
\begin{aligned}
\lim _{\left\langle y_{\alpha}^{2}\right\rangle \rightarrow 0} \mathscr{C}_{\alpha \alpha} & =\lim _{\left\langle y_{\alpha}^{2}\right\rangle \rightarrow 0}\left\langle y_{\alpha}^{2}\right\rangle_{t_{t}} \geq 0, \\
\lim _{\left\langle y_{\alpha}^{2}\right\rangle \rightarrow 1} \mathscr{C}_{\alpha \alpha} & =\lim _{\left\langle y_{\alpha}^{2}\right\rangle \rightarrow 1}\left\langle y_{\alpha}^{2}\right\rangle_{t_{t}} \leq 0,
\end{aligned}
$$

as the boundary of the state space is approached, indicating that in general the equation governing $\left\langle y_{\alpha}^{2}\right\rangle$ must either be a function of $\left\langle y_{\alpha}^{2}\right\rangle$ or $\mathscr{C}_{\alpha \alpha} \equiv 0$ for all $t$.

(3) Boundedness of the covariances, (11). This condition can be ensured if, for $\alpha \neq \beta$,

$$
\begin{aligned}
\lim _{\left\langle y_{\alpha} y_{\beta}\right\rangle \rightarrow-1} \mathscr{C}_{\alpha \beta} & =\lim _{\left\langle y_{\alpha} y_{\beta}\right\rangle \rightarrow-1}\left\langle y_{\alpha} y_{\beta}\right\rangle_{t_{t}} \geq 0, \\
\lim _{\left\langle y_{\alpha} y_{\beta}\right\rangle \rightarrow 1} \mathscr{C}_{\alpha \beta} & =\lim _{\left\langle y_{\alpha} y_{\beta}\right\rangle \rightarrow 1}\left\langle y_{\alpha} y_{\beta}\right\rangle_{{ }_{t}} \leq 0,
\end{aligned}
$$

as the boundary of the state space is approached, indicating that in general the equation governing $\left\langle y_{\alpha} y_{\beta}\right\rangle$ must either be a function of $\left\langle y_{\alpha} y_{\beta}\right\rangle$ or $\mathscr{C}_{\alpha \beta} \equiv 0$ for all $t$.

(4) Zero row sums, (12). Differentiating (12) in time and using (31) yield the system

$$
\begin{gathered}
\mathscr{C}_{11}+\mathscr{C}_{21}+\cdots+\left\langle y_{N} y_{1}\right\rangle_{t}=0 \\
\mathscr{C}_{12}+\mathscr{C}_{22}+\cdots+\left\langle y_{N} y_{2}\right\rangle_{t}=0 \\
\vdots \\
\mathscr{C}_{1(N-1)}+\mathscr{C}_{2(N-1)}+\cdots+\left\langle y_{N} y_{N-1}\right\rangle_{t}=0 \\
\left\langle y_{1} y_{N}\right\rangle_{t_{t}}+\left\langle y_{2} y_{N}\right\rangle_{t_{t}}+\cdots+\left\langle y_{N}^{2}\right\rangle_{t_{t}}=0 .
\end{gathered}
$$

Performing the same substitutions on (35) that resulted in (13) we obtain the weaker constraint:

$$
\sum_{\alpha=1}^{N-1} \sum_{\beta=1}^{N-1} \mathscr{C}_{\alpha \beta}-\left\langle y_{N}^{2}\right\rangle{ }_{t}=0 .
$$

We see that the trivial specification, $\mathscr{C}_{\alpha \beta} \equiv 0$, satisfies all the above conditions but also fixes the covariance matrix at its initial state for all $t \geq t_{0}$, which is of limited applicability.

4.3. Bounded Evolution of the Third Central Moments, $\left\langle y_{\alpha}^{3}\right\rangle$. Multiplying the Fokker-Planck equation (17) by $y_{\gamma}^{3}=$ $\left(Y_{\gamma}-\left\langle Y_{\gamma}\right\rangle\right)^{3}$ and then integrating yield the system governing the third central moments, $\left\langle y_{\alpha}^{3}\right\rangle$, as

$$
\begin{array}{r}
\frac{\partial\left\langle y_{\alpha}^{3}\right\rangle}{\partial t}=3\left\langle y_{\alpha}^{2} A_{\alpha}\right\rangle+3 \sum_{\beta=1}^{N-1}\left\langle y_{\alpha} B_{\beta \beta}\right\rangle=\mathcal{S}_{\alpha} \\
\alpha=1, \ldots, N-1,
\end{array}
$$

with $A_{\alpha}=A_{\alpha}(\mathbf{Y}, t)$ and $B_{\alpha \beta}=B_{\alpha \beta}(\mathbf{Y}, t)$. The right hand sides of (37) are the evolution rates of the third moments, denoted by $\mathcal{S}_{\alpha}$. The boundedness of the third moments, required by (14), can be ensured if

$$
\begin{aligned}
\lim _{\left\langle y_{\alpha}^{3}\right\rangle \rightarrow-1} \mathcal{S}_{\alpha} & =\lim _{\left\langle y_{\alpha}^{3}\right\rangle \rightarrow-1}\left\langle y_{\alpha}^{3}\right\rangle_{{ }_{t}} \geq 0 \\
\lim _{\left\langle y_{\alpha}^{3}\right\rangle \rightarrow 1} \mathcal{S}_{\alpha} & =\lim _{\left\langle y_{\alpha}^{3}\right\rangle \rightarrow 1}\left\langle y_{\alpha}^{3}\right\rangle_{{ }_{t}} \leq 0
\end{aligned}
$$

as the boundary of the state space is approached, indicating that in general the equation governing $\left\langle y_{\alpha}^{3}\right\rangle$ must either be a function of $\left\langle y_{\alpha}^{3}\right\rangle$ or $\mathcal{S}_{\alpha} \equiv 0$ for all $t$. The conditions in (38) only ensure boundedness; consequently, they are necessary but not sufficient conditions for realizability of the third moments as required by (2). Note that the requirement on bounded sample space has no implications on the boundedness of the skewness:

$$
-\infty<\frac{\left\langle y_{\alpha}^{3}\right\rangle}{\left\langle y_{\alpha}^{2}\right\rangle^{3 / 2}}<\infty,
$$

since $\left\langle y_{\alpha}^{2}\right\rangle \geq 0$, see $(10)$.

4.4. Bounded Evolution of the Fourth Central Moments, $\left\langle y_{\alpha}^{4}\right\rangle$. Multiplying the Fokker-Planck equation (17) by $y_{\gamma}^{4}=$ $\left(Y_{\gamma}-\left\langle Y_{\gamma}\right\rangle\right)^{4}$ and then integrating yield the system governing the fourth central moments, $\left\langle y_{\alpha}^{4}\right\rangle$, as

$$
\begin{array}{r}
\frac{\partial\left\langle y_{\alpha}^{4}\right\rangle}{\partial t}=4\left\langle y_{\alpha}^{3} A_{\alpha}\right\rangle+6 \sum_{\beta=1}^{N-1}\left\langle y_{\alpha}^{2} B_{\beta \beta}\right\rangle=\mathscr{K}_{\alpha}, \\
\alpha=1, \ldots, N-1,
\end{array}
$$


with $A_{\alpha}=A_{\alpha}(\mathbf{Y}, t)$ and $B_{\alpha \beta}=B_{\alpha \beta}(\mathbf{Y}, t)$. The right hand sides of (40) are the evolution rates of the fourth moments, denoted by $\mathscr{K}_{\alpha}$. The boundedness of the fourth moments, required by (15), can be ensured if

$$
\begin{aligned}
& \lim _{\left\langle y_{\alpha}^{4}\right\rangle \rightarrow 0} \mathscr{K}_{\alpha}=\lim _{\left\langle y_{\alpha}^{4}\right\rangle \rightarrow 0}\left\langle y_{\alpha}^{4}\right\rangle_{{ }_{t}} \geq 0, \\
& \lim _{\left\langle y_{\alpha}^{4}\right\rangle \rightarrow 1} \mathscr{K}_{\alpha}=\lim _{\left\langle y_{\alpha}^{4}\right\rangle \rightarrow 1}\left\langle y_{\alpha}^{4}\right\rangle_{{ }_{t}} \leq 0,
\end{aligned}
$$

as the boundary of the state space is approached, indicating that in general the equation governing $\left\langle y_{\alpha}^{4}\right\rangle$ must either be a function of $\left\langle y_{\alpha}^{4}\right\rangle$ or $\mathscr{K}_{\alpha} \equiv 0$ for all $t$. The conditions in (41) only ensure boundedness; consequently, they are necessary but not sufficient conditions for realizability of the fourth moments as required by (2). Note that, similar to the skewness in (39), the requirement on bounded sample space has no implications on the upper bound of the kurtosis:

$$
0 \leq \frac{\left\langle y_{\alpha}^{4}\right\rangle}{\left\langle y_{\alpha}^{2}\right\rangle^{2}}<\infty,
$$

since $\left\langle y_{\alpha}^{2}\right\rangle \geq 0,(10)$.

4.5. Summary on Realizable Statistics of Fractions. The unitsum constraint, (4), applied to a set of nonnegative random variables, bounds and constrains their statistical moments, as shown in Section 2.2, as well as their time evolutions. We examined the evolution of the moments, $\left\langle Y_{\alpha}\right\rangle,\left\langle y_{\alpha} y_{\beta}\right\rangle,\left\langle y_{\alpha}^{3}\right\rangle$, and $\left\langle y_{\alpha}^{4}\right\rangle$, and showed how they are governed if an underlying diffusion process is known.

Realizability of the means, as defined by (2), can be ensured if (8) and (30) are satisfied. Realizability of the covariances can be ensured if (10)-(12) and (32)-(35) are satisfied. Boundedness of the third moments is ensured by (14) and (38), while boundedness of the fourth moments is ensured by (15) and (41). The procedure outlined above can be continued to derive additional constraints for consistency of the third, fourth, mixed, and higher moments with the unit-sum constraint. The constraints reflect the coupled and nonlinear nature of random fractions, both as instantaneous variables and their statistics.

\section{A Survey of Realizable Diffusion Processes}

A survey of existing diffusion processes that satisfy the realizability constraints on drift and diffusion on the statespace boundary, (28), is now given.

5.1. Realizable Binary Process: $N=2$, Beta. An example for $N=2$, satisfying the realizability constraints on the drift and diffusion terms on the sample-space boundary in (23), is given in [20], specifying the drift and diffusion as

$$
\begin{aligned}
& A(Y)=\frac{b}{2}(S-Y), \\
& B(Y)=\kappa Y(1-Y),
\end{aligned}
$$

yielding the stochastic differential equation:

$$
\mathrm{d} Y(t)=\frac{b}{2}(S-Y) \mathrm{d} t+\sqrt{\kappa Y(1-Y)} \mathrm{d} W(t),
$$

with $b>0, \kappa>0$, and $0<S<1$ excluding, while with $0 \leq$ $S \leq 1$ allowing for absorbing barriers. In (44) the drift is linear and the diffusion is quadratic in $Y$. The invariant distribution of (44) is beta, which belongs to the family of Pearson distributions, discussed in detail by Forman \& Sørensen [21]. Of the special cases of the Pearson diffusions, discussed in [21], only Case 6, equivalent to (44), produces realizable events. A symmetric variant of (44) was constructed in [22], which does not allow a nonzero skewness in the statistically stationary state; see [20].

5.2. Realizable Multivariate Process: $N>2$, Wright-Fisher. A system of stochastic differential equations that satisfies the realizability conditions for $N>2$ variables in (28) is the multivariate Wright-Fisher process [9], which specifies the drift and diffusion terms as

$$
\begin{aligned}
& A_{\alpha}(\mathbf{Y})=\frac{1}{2}\left(\omega_{\alpha}-\omega Y_{\alpha}\right), \\
& B_{\alpha \beta}(\mathbf{Y})=Y_{\alpha}\left(\delta_{\alpha \beta}-Y_{\beta}\right),
\end{aligned}
$$

yielding the stochastic process,

$$
\begin{array}{r}
\mathrm{d} Y_{\alpha}(t)=\frac{1}{2}\left(\omega_{\alpha}-\omega Y_{\alpha}\right) \mathrm{d} t+\sum_{\beta=1}^{N-1} \sqrt{Y_{\alpha}\left(\delta_{\alpha \beta}-Y_{\beta}\right)} \mathrm{d} W_{\alpha \beta}(t), \\
\alpha=1, \ldots, N-1,
\end{array}
$$

where $\omega=\sum_{\beta=1}^{N} \omega_{\beta}$ and $\omega_{\beta}>0$ are parameters. Equation (46) is a generalization of (44) for $N>2$ variables. The invariant distribution of (46) is Dirichlet $[23,24]$.

5.3. Realizable Multivariate Process: $N>2$, Dirichlet. Another process that satisfies (28), developed in [24], specifies the drift and diffusion terms as

$$
\begin{aligned}
& A_{\alpha}(\mathbf{Y})=\frac{b_{\alpha}}{2}\left[S_{\alpha} Y_{N}-\left(1-S_{\alpha}\right) Y_{\alpha}\right], \\
& B_{\alpha \beta}(\mathbf{Y})= \begin{cases}\kappa_{\alpha} Y_{\alpha} Y_{N} & \text { for } \alpha=\beta, \\
0 & \text { for } \alpha \neq \beta,\end{cases}
\end{aligned}
$$

resulting in the system of stochastic differential equations,

$$
\begin{array}{r}
\mathrm{d} Y_{\alpha}(t)=\frac{b_{\alpha}}{2}\left[S_{\alpha} Y_{N}-\left(1-S_{\alpha}\right) Y_{\alpha}\right] \mathrm{d} t+\sqrt{\kappa_{\alpha} Y_{\alpha} Y_{N}} \mathrm{~d} W_{\alpha}(t), \\
\alpha=1, \ldots, N-1,
\end{array}
$$

with parameter vectors $b_{\alpha}>0, \kappa_{\alpha}>0$, and $0<S_{\alpha}<1$, and $Y_{N}$ given by (6). Equation (48) is also a generalization of (44) 
for $N>2$ variables. The invariant distribution of (48) is also Dirichlet, provided the parameters of the drift and diffusion terms satisfy

$$
\left(1-S_{1}\right) \frac{b_{1}}{\kappa_{1}}=\cdots=\left(1-S_{N-1}\right) \frac{b_{N-1}}{\kappa_{N-1}} .
$$

Note that while there is no coupling among the parameters, $\omega_{\alpha}$, of the drift and diffusion terms in the Wright-Fisher equation (46), the parameters, $b_{\alpha}, S_{\alpha}$, and $\kappa_{\alpha}$, of (48) must be constrained by (49) to keep its invariant distribution Dirichlet.

5.4. Realizable Multivariate Process: $N>2$, Lochner's Generalized Dirichlet. A generalization of (48) is developed in [25], where the drift and diffusion terms are given by

$$
\begin{gathered}
A_{\alpha}(\mathbf{Y})=\frac{\mathscr{U}_{\alpha}}{2}\left\{b_{\alpha}\left[S_{\alpha} \mathscr{Y}_{K}-\left(1-S_{\alpha}\right) Y_{\alpha}\right]+Y_{\alpha} \mathscr{Y}_{K} \sum_{\beta=\alpha}^{K-1} \frac{c_{\alpha \beta}}{\mathcal{Y}_{\beta}}\right\}, \\
B_{\alpha \beta}(\mathbf{Y})= \begin{cases}\kappa_{\alpha} Y_{\alpha} \mathscr{Y}_{K} \mathscr{U}_{\alpha} & \text { for } \alpha=\beta, \\
0 & \text { for } \alpha \neq \beta,\end{cases}
\end{gathered}
$$

with $\mathscr{Y}_{\alpha}=1-\sum_{\beta=1}^{\alpha} Y_{\beta}$ and $\mathscr{U}_{\alpha}=\prod_{\beta=1}^{K-\alpha} \mathscr{Y}_{K-\beta}^{-1}$, yielding the stochastic process,

$$
\begin{gathered}
\mathrm{d} Y_{\alpha}(t)=\frac{\mathscr{U}_{\alpha}}{2}\left\{b_{\alpha}\left[S_{\alpha} \mathscr{Y}_{K}-\left(1-S_{\alpha}\right) Y_{\alpha}\right]\right. \\
\left.+Y_{\alpha} \mathscr{Y}_{K} \sum_{\beta=\alpha}^{K-1} \frac{c_{\alpha \beta}}{\mathscr{Y}_{\beta}}\right\} \mathrm{d} t \\
+\sqrt{\kappa_{\alpha} Y_{\alpha} \mathscr{Y}_{K} \mathscr{U}_{\alpha}} \mathrm{d} W_{\alpha}(t), \\
\alpha=1, \ldots, K=N-1 .
\end{gathered}
$$

The invariant distribution of (51) is Lochner's generalized Dirichlet distribution [26], if the coefficients, $b_{\alpha}>0, \kappa_{\alpha}>0$, $0<S_{\alpha}<1$, and $c_{\alpha \beta}$, with $c_{\alpha \beta}=0$ for $\alpha>\beta, \alpha, \beta=1, \ldots, K-1$, satisfy the conditions developed in [25]. Similar to (48), the parameters of the drift and diffusion terms, $b_{\alpha}, S_{\alpha}, \kappa_{\alpha}$, and $c_{\alpha \beta}$, of (51) must be constrained to keep the invariant distribution generalized Dirichlet. Setting

$$
\frac{c_{1 i}}{\kappa_{i}}=\cdots=\frac{c_{i i}}{\kappa_{i}}=1 \quad \text { for } i=1, \ldots, K-1,
$$

in (51) reduces to the standard Dirichlet process, (48).

All of (46), (48), and (51) have coupled and nonlinear diffusions terms. As discussed earlier, this is required to simultaneously satisfy the realizability conditions in (28), required to represent $N>2$ random fractions by diffusion processes.

\section{Summary}

We have demonstrated that the problem of $N$ fluctuating variables constrained by the unit-sum requirement can be discussed in a reduced sample space of $N-1$ dimensions. This allows working with the unique, universal, and mathematically well-defined realizable sample space which produces samples and statistics consistent with the underlying conservation principle.

We have studied multivariate diffusion processes governing a set of fluctuating variables required to satisfy two constraints: (1) nonnegativity and (2) a conservation principle that requires the variables to sum to one, defined as realizability. Our findings can be summarized as follows.

(i) The diffusion coefficients in stochastic diffusion processes, governing fractions, must be coupled and nonlinear.

(ii) If the set of constraints,

$$
\begin{gathered}
A_{\alpha}\left(Y_{\alpha}=0, Y_{\beta \neq \alpha}, t\right) \geq 0, \\
B_{\alpha \beta}\left(Y_{\alpha}=0, Y_{\beta \neq \alpha}, t\right)=0, \\
A_{\alpha}\left(\sum_{\alpha=1}^{N-1} Y_{\alpha}=1, t\right) \leq 0, \\
B_{\alpha \beta}\left(\sum_{\alpha=1}^{N-1} Y_{\alpha}=1, t\right)=0, \\
\alpha, \beta=1, \ldots, N-1,
\end{gathered}
$$

is satisfied as the state-space boundary is approached, the stochastic system,

$$
\begin{aligned}
\mathrm{d} Y_{\alpha}(t) & =A_{\alpha}(\mathbf{Y}, t) \mathrm{d} t+\sum_{\beta=1}^{N-1} b_{\alpha \beta}(\mathbf{Y}, t) \mathrm{d} W_{\beta}(t), \\
& \alpha=1, \ldots, N-1, \\
Y_{N} & =1-\sum_{\alpha=1}^{N-1} Y_{\alpha},
\end{aligned}
$$

with $B_{\alpha \beta}=\sum_{\gamma=1}^{N-1} b_{\alpha \gamma} b_{\gamma \beta}$, ensures that the components of the vector of fractions, $\mathbf{Y}=\left(Y_{1}, \ldots, Y_{N}\right)$, remain nonnegative and sum to one at all times.

(iii) Boundedness of the sample space requires boundedness of the moments.

The constraints provide a method that can be used to develop drift and diffusion functions for stochastic diffusion processes for variables satisfying a conservation law and thus are inherently realizable.

\section{Conflict of Interests}

The authors declare that there is no conflict of interests regarding the publication of this paper.

\section{References}

[1] K. Pearson, "Mathematical contributions to the theory of evolution. On a form of spurious correlation which may arise 
when indices are used in the measurement of organs," Royal Society of London Proceedings I, vol. 60, pp. 489-498, 1896.

[2] C. D. M. Paulino and C. A. D. B. Pereira, "Bayesian methods for categorical data under informative general censoring," Biometrika, vol. 82, no. 2, pp. 439-446, 1995.

[3] F. Chayes, "Numerical correlation and petrographic variation," The Journal of Geology, vol. 70, no. 4, pp. 440-452, 1962.

[4] F. Chayes and W. Kruskal, "An approximate statistical test for correlations between proportions," The Journal of Geology, vol. 74, no. 5, pp. 692-702, 1966.

[5] P. S. Martin and J. E. Mosimann, "Geochronology of pluvial Lake Cochise, Southern Arizona, [part] 3, Pollen statistics and Pleistocene metastability," American Journal of Science, vol. 263, pp. 313-358, 1965.

[6] K. Lange, "Applications of the Dirichlet distribution to forensic match probabilities," Genetica, vol. 96, no. 1-2, pp. 107-117, 1995.

[7] C. Gourieroux and J. Jasiak, "Multivariate Jacobi process with application to smooth transitions," Journal of Econometrics, vol. 131, no. 1-2, pp. 475-505, 2006.

[8] S. S. Girimaji, "Assumed $\beta$-pdf model for turbulent mixing: validation and extension to multiple scalar mixing," Combustion Science and Technology, vol. 78, no. 4, pp. 177-196, 1991.

[9] M. Steinrücken, Y. X. R. Wang, and Y. S. Song, "An explicit transition density expansion for a multi-allelic Wright-Fisher diffusion with general diploid selection," Theoretical Population Biology, vol. 83, pp. 1-14, 2013.

[10] J. G. Mauldon, "Random division of an interval," Mathematical Proceedings of the Cambridge Philosophical Society, vol. 47, no. 2, pp. 331-336, 1951.

[11] J. G. Mauldon, "A generalization of the beta-distribution," The Annals of Mathematical Statistics, vol. 30, no. 2, pp. 509-520, 1959.

[12] I. J. Good, The Estimation of Probabilities, Number 30 in Research Monograph, The MIT Press, Cambridge, Mass, USA, 1965.

[13] R. Pyke, "Spacings," Journal of the Royal Statistical Society B, vol. 27, no. 3, pp. 395-449, 1965.

[14] C. W. Gardiner, Stochastic Methods, A Handbook for the Natural and Social Sciences, Springer, Berlin, Germany, 4th edition, 2009.

[15] P. E. Kloeden and E. Platen, Numerical Solution of Stochastic Differential Equations, Springer, Berlin, Germany, 1999.

[16] S. B. Pope, "Accessed compositions in turbulent reactive flows," Flow, Turbulence and Combustion, vol. 72, no. 2-4, pp. 219-243, 2004.

[17] N. G. van Kampen, Stochastic Processes in Physics and Chemistry, North Holland, Elsevier B. V., Amsterdam, The Netherlands, 2nd edition, 2004.

[18] W. Feller, "The parabolic differential equations and the associated semi-groups of transformations," Annals of Mathematics, vol. 55, no. 3, pp. 468-519, 1952.

[19] S. B. Pope, "PDF methods for turbulent reactive flows," Progress in Energy and Combustion Science, vol. 11, no. 2, pp. 119-192, 1985.

[20] J. Bakosi and J. R. Ristorcelli, "Exploring the beta distribution in variable-density turbulent mixing," Journal of Turbulence, vol. 11, no. 37, pp. 1-31, 2010.

[21] J. L. Forman and M. Sørensen, "The Pearson diffusions: a class of statistically tractable diffusion processes," Scandinavian Journal of Statistics, vol. 35, no. 3, pp. 438-465, 2008.
[22] G. Q. Cai and Y. K. Lin, "Generation of non-Gaussian stationary stochastic processes," Physical Review E, vol. 54, no. 1, pp. 299303, 1996.

[23] S. Wright, "Adaptation and selection," in Genetics, Paleontology, and Evolution, E. Mayr, G. L. Jepson, and G. G. Simpson, Eds., pp. 365-389, Princeton University Press, 1949.

[24] J. Bakosi and J. R. Ristorcelli, "A stochastic diffusion process for the Dirichlet distribution," International Journal of Stochastic Analysis, vol. 2013, Article ID 842981, 7 pages, 2013.

[25] J. Bakosi and J. R. Ristorcelli, "A stochastic diffusion process for Lochner's generalized Dirichlet distribution," Journal of Mathematical Physics, vol. 54, no. 10, Article ID 102701, 2013.

[26] R. H. Lochner, "A generalized Dirichlet distribution in Bayesian life testing," Journal of the Royal Statistical Society B, vol. 37, no. 1, pp. 103-113, 1975. 


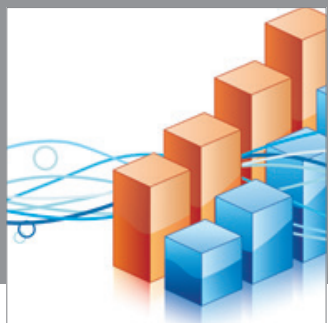

Advances in

Operations Research

mansans

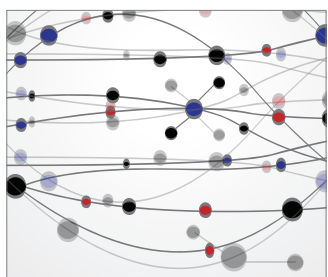

The Scientific World Journal
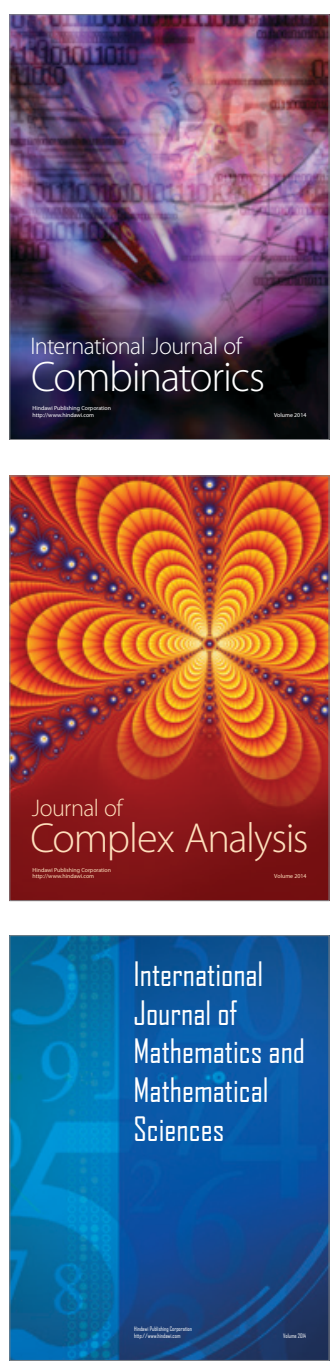
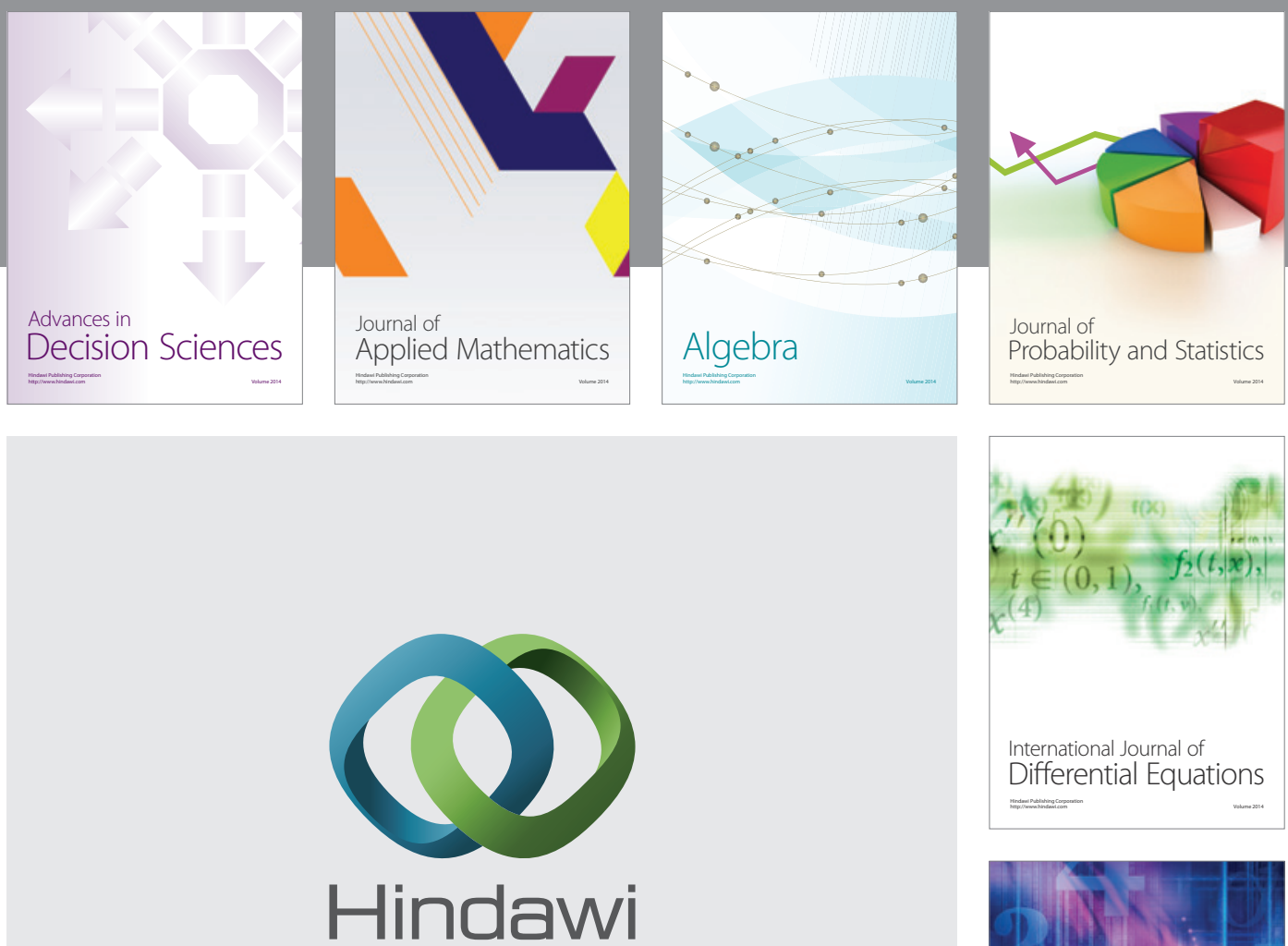

Submit your manuscripts at http://www.hindawi.com
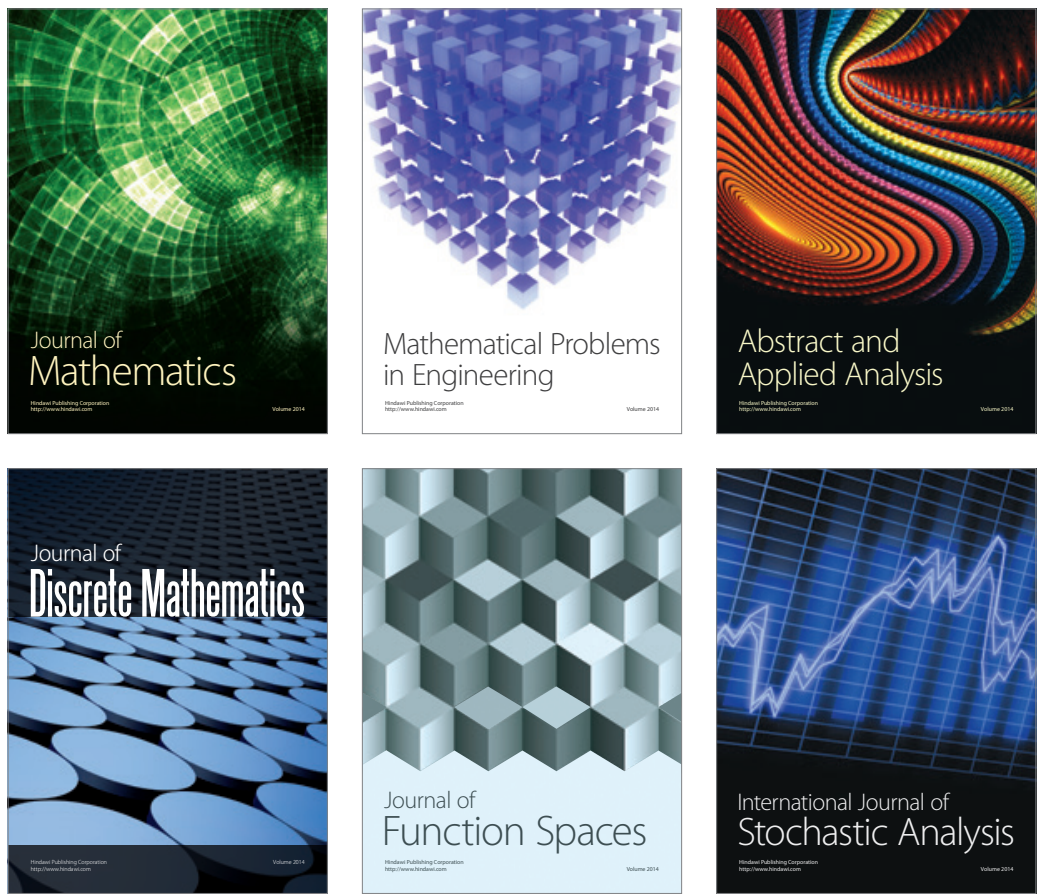

Journal of

Function Spaces



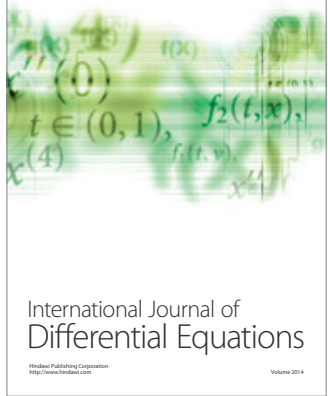
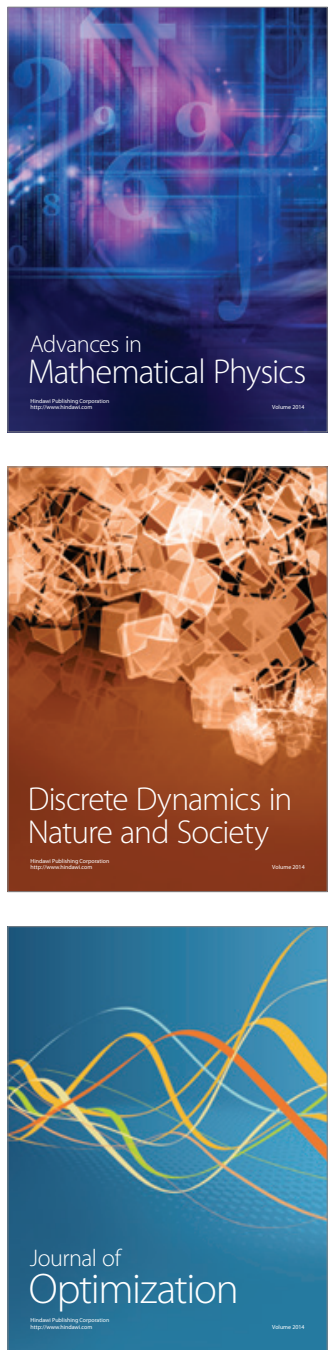